\title{
PSS Design Based on Fuzzy Controller with Particle Swarm Optimization Tuning by Ermanu Azizul Hakim
}

Submission date: 05-Aug-2020 12:02PM (UTC+0700)

Submission ID: 1366107623

File name: PID5587617.pdf (247.21K)

Word count: 2090

Character count: 10543 


\section{PSS Design Based on Fuzzy Controller with Particle Swarm Optimization Tuning}

\author{
Ermanu A. Hakim \\ Electrical Engineering Department \\ Universitas Muhammadiyah Malang \\ Malang, Indonesia \\ ermanu@umm.ac.id
}

\author{
Nur Kasan \\ Electrical Engineering Department \\ Universitas Muhammadiyah Malang \\ Malang, Indonesia \\ kasan@umm.ac.id
}

\author{
Nurhadi \\ Electrical Engineering Department \\ Universitas Muhammadiyah Malang \\ Malang, Indonesia \\ nurhadi@umm.ac.id
}

\begin{abstract}
The research was conducted for implementating of PSS (Power system stabilizer) which was designed based on the fuzzy logic controller (FLPSS). This approach has a main purpose for stabilizing and improving the damping of synchronous machine. The speed and active power deviation were used as fuzzy controller's inputs. The controller's output was forwarded into AVR subsequently. In order to achieve optimal setting, the optimal criteria of the Integral of Time were multiplied by the Absolute Error (ITAE). The performance of the proposed PSS under small disturbances, system parameters and loading conditions was tested. The experiment's results showed the usefulness of the specified method for damping out the system oscillations.
\end{abstract}

Key words - Fuzzy Logic Controller, Particle Swarm Optimization, power system stabilizer, synchronous machine, optimal stability,

\section{INTRODUCTION}

Low frequency oscillation is a common problem in power system operations. PSS (power system stabilizer) is one alternative solution to this problem. PSS can provide additional control signals for the excitation system or speed control system of the power generation unit. It can also reduce oscillations and improve dynamic performance. PSS has been extensively studied and used in power systems for many years.

Most PSS implementation comprehends the classical linear control paradigm. The PSS method is based on a linear model in a power system's default configuration. This results in a fixed parameter of PSS. This is called conventional PSS (CPSS) and is prominently used in power systems to reduce small oscillations. [1-3].

In conventional fixed-parameter spectrum controllers, gain and other parameters may not correspond to the entire spectrum of operations. Along with the growth of technological innovation, it become feasible for developing and establishing improved controllers based on a variety of sophisticated modern techniques. Power system stabilizers based on fuzzy logic, adaptive controls and artificial neural networks are being developed. Each of these control techniques has unique features and strengths. Fuzzy logicbased PSS (FLPSS) showed a significant possibility in term of increasing generator oscillation damping [6].

In this paper, fuzzy based PSS was implemented using the speed deviation and active power deviation as an inputs.

\section{POWER SYSTEM MODFL}

The proposed approach considered a single machine infinite bus (SMIB) system with synchronous generator provided with IEEE type-ST1 static excitation system. Fig. 1 [2] illustrates the model of the power system in linear manner. The nominal operating conditions and system parameters are given.

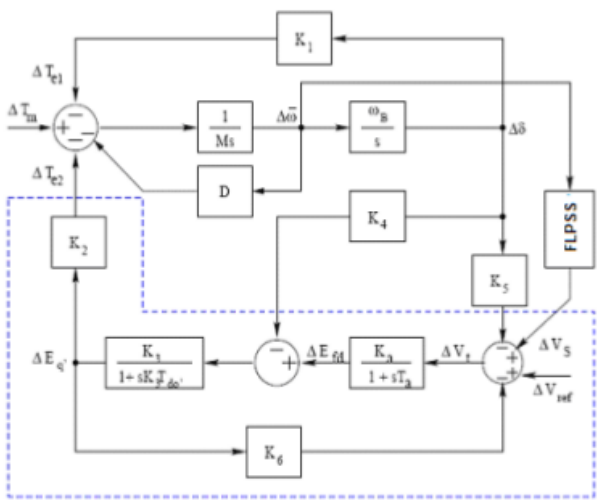

Fig. 1 A linear model of power system

System Data:

Generator:

$M=2 H=7 ; X_{d}=1,81 ; X_{q}=1,76 ; X_{d}^{\prime}=0,3$

$L_{a d u}=1,65 ; L_{a q u}=1,60 ; L_{I}=0,16$

$R_{a}=0,03 ; R_{f d}=0,0006 ; L_{f d}=0,153$

$A_{\text {sat }}=0,031 ; B_{\text {sat }}=6,93 \psi_{T 7}=0,8$

Excitation System:

$K_{A V R}=400 ; T_{A}=0,05 ; T_{B}=1,0 ; T_{C}=8,0 ; T_{R}=0,02$

PSS:

$T_{W}=1,4$

Transmition Line:

$X_{e}=0,65 ; R_{e}=0,0$

Operation Condition: 


\section{FUZZY LOGIC BASED PSS}

PSS based on Fuzzy logic controller algorithm has been developed. The main objective of the proposed application was intended for stabilizing and improving the damping of the synchronous machine [4]. A speed $\Delta \omega$ and active power deviation $\Delta \mathrm{P}_{\mathrm{e}}$ are pointed as the controller input. The controller output is forwarded into the exciter module.

As can be seen in Fig. 2, the fuzzy-logic based PSS uses two input parameters including $K_{\omega}$ and $K_{\mathrm{p}}$, whereas the output parameter, $K_{\mathrm{u}}$. The input parameters selection process is generally subjective which demands the previous knowledge of fuzzy control variables (input and output signal).

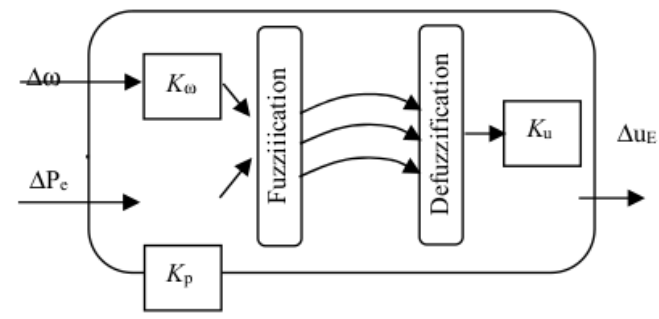

Fig. 2. Schematic diagram of the FLPSS

Using the automatic rule generation and sampled data set generated by using the conventional power system stabilizer, a proper set of rules was obtained [4]. The rule used in all the following are shown in figure 3 .

\begin{tabular}{|l|l|l|l|l|l|l|l|l|}
\hline & \multicolumn{7}{|c|}{ Active power } \\
\hline \multirow{3}{*}{} & & NB & NM & NS & Z & PS & PM & PB \\
\cline { 2 - 9 } & NB & NB & NB & NB & NB & NM & NS & Z \\
\cline { 2 - 9 } & NM & NB & NB & NM & NM & NS & Z & PS \\
\cline { 2 - 9 } & NS & NB & NM & NS & NS & Z & PS & PM \\
\cline { 2 - 9 } & Z & NM & NM & NS & Z & PS & PM & PM \\
\cline { 2 - 8 } & PS & NM & NS & Z & PS & PS & PM & PB \\
\cline { 2 - 9 } & PM & NS & Z & PS & PM & PM & PB & PB \\
\cline { 2 - 8 } & PB & Z & PS & PM & PB & PB & PB & PB \\
\hline
\end{tabular}

Fig. 3. FLPSS rules

\section{PARTICLE SWARM OPTIMIZATION}

In 1995, Kennedy and Eberhart became the pioneers for introducing the Particle swarm optimization (PSO) method [8]. Subsequently, the original Swarm algorithm was modified for improving the performance and adjusting to particular types of problems, the serial version has been implemented previously [9]-[11].

The following are the brief stages of optimization using the PSO algorithm [11]. Consider a swarm of $p$ particles, with each particle position describing the possible solution points in the space of the design problem of $\mathrm{D}$. The position $x_{i}$ is updated upon each of particle based on the following notation:

$$
x_{k+1}^{i}=x_{k}^{i}+v_{k+1}^{i}
$$

with a pseudo-velocity $\boldsymbol{v}_{k+1}^{i}$ can be calculated as follows:

$v_{k+1}^{i}=\omega_{k} v_{k}^{i}+c_{1} r_{1}\left(p_{k}^{i}-x_{k}^{i}\right)+c_{2} r_{2}\left(p_{k}^{g}-x_{k}^{i}\right)$

Here, subscript $k$ denote a 2 hit) pseudo-time increment, $\mathbf{p}_{k}^{i}$ denotes the best position of particle $i$ at time $k$ (the cognitive contribution to the pseudo-velocity vector $v_{k+1}^{i}$ ), while $\mathbf{p}_{k}^{g}$ symbolizes the best global position in the swarm at time $k$ (social contribution). $r_{l}$ and $r_{2}$ denotes uniform random numbers between 0 and 1 . The cognitive and social scaling parameters $c_{1}$ and $c_{2}$ are systematically selected such that $c_{l}=c_{2}=2$ for allowing the product $c_{l} r_{l}$ or $c_{2} r_{2}$ to have a mean of 1 .

The particles overshoot the target half the time is the result of using these proposed values which maintains the separation within the group and if there is no overshoot occurred, it will allow for a greater area to be searched.

Fourie and Groenwold generated a comprehensive modification in the original PSO algorithm which permits the transition for searching another result as optimization progresses. This operator decreases the maximum allowed velocity $v_{\max } k$ and 1 the particle inertia $w_{k}$ in a dynamic manner, as directed by the dynamic reduction parameters $\mathrm{k}$, $\mathrm{d}, \omega$. For the sake of brevity, further details of this operator are neglected.

The serial 250 algorithm was performed on a single CPU computer. The total number of particles in 11e swarm is symbolized by the variable of $p$. The significant fitness value from a particular particle at design co-ordinates $\mathbf{p}_{k}^{i}$ is presented by $f_{\text {best }}^{i}$ and the best fitness value in the overall swarm at co-ordinates $p_{k}^{g}$ by $f_{\text {best }}^{g}$. At $k=0$, the particle velocities $v_{0}^{i} . v_{0}^{\text {maks }}$ has its initial value resided within the limits of $0 \leq v_{0} \leq v_{0}^{\text {maks }}$. The vector $v_{0}^{\text {maks }}$ is extracted from the fraction of the distance between the upper and lower bounds $v_{0}^{\text {maks }}=\zeta\left(\mathbf{x}_{U B}-\mathbf{x}_{\text {LB }}\right)$ with $\zeta=0.5$. The details of PSO algorithm is expounded as follows.

1. Initialize

(a) Set values constants $\mathrm{k}_{\text {maks }}, \mathrm{c}_{1}, \mathrm{c}_{2}, \mathrm{k}, v_{0}^{\text {maks }}, \mathrm{w}_{\mathrm{k}}, \mathrm{d}$, and $w d$

(b) Initialize the maximum dynamic velocity $v_{\max }, k$ and inertia $w k$

(c) Set counters $k=0, t=0, i=1$. Set random number seed

(d) initialize randomly particle positions $\mathbf{x}_{\mathbf{i}} \in \mathbf{D}$ in $\mathrm{R}_{\mathrm{n}}$ for $i=1, \ldots, p$

(e) initialize randomly particle velocities $0 \leq v_{0} \leq v_{0}^{\text {maks }}$ for $i=1, \ldots, p$

(f) Evaluate fitness values $f_{0}^{i}$ using design space coordinates $\mathbf{x}_{\mathbf{i} 0}$ for $i=1, \ldots, p$ 
(g) Set $f_{\text {best }}^{i}=f_{0}^{i}, \mathbf{p}^{i}=\mathbf{x}_{0}^{i}$ for $i=1, \ldots, p$

(h) Set $f_{\text {best }}^{g}$ to $f_{\text {best }}^{i}$ and $\mathbf{g}_{0}$ to corresponding $\mathbf{x}_{0}^{i}$

2. Optimize

(a) Update the particle of velocity vector $\mathbf{v}_{k+1}^{i}$ using Equation (2)

(b) Update the particle of position vector $\mathbf{x}_{k+1}^{i}$ using Equation (1)

(c) Update the maximum dynamic velocity $\mathbf{v}_{k}^{m a k s}$ and inertia $w k$

(d) Evaluate the fitness value $f_{k}^{i}$ using design space co-ordinates $\mathbf{x}_{k}^{i}$

(e) if $f_{k}^{i} \leq f_{\text {best }}^{i}$, then $f_{\text {best }}^{i}=f_{k}^{i}, \mathbf{p}^{i}=\mathbf{x}_{k}^{i}$

(f) if $f_{k}^{i} \leq f_{\text {best } 1}^{g}$ then $f_{\text {best }}^{g}=f_{k}^{i}, \mathbf{p}^{g}=\mathbf{x}_{k}^{i}$

(g) if $f_{\text {best }}^{g}$ best was improved in (e) then reset $t=0$. Else increment $t$

(h) $2 \mathrm{k}>\mathrm{k}_{\text {maks }}$ gp to 3

(i) if $\mathrm{t}=\mathrm{d}$ then multiply $w_{k+1}$ by $\left(1-\mathrm{w}_{\mathrm{d}}\right)$ and $v_{k+1}^{m a k s}$ by $\left(1-v_{d}\right)$

(j) 11 stopping condition is fulfilled, then moves to 3

(k) Increment $\mathrm{i}$, if $\mathrm{i}>\mathrm{p}$ then increment $k$, and set $i=1$

(l) Go to 2(a)

3. Report results

4. Terminate

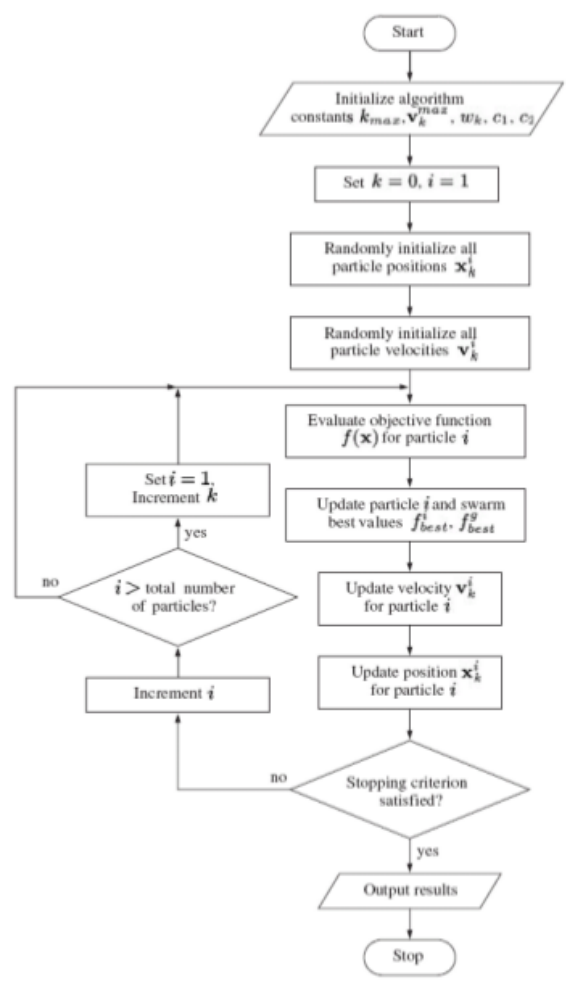

Fig. 4. Serial PSO implementation ${ }^{11}$

\section{SIMULATION RESULT}

In order to analyze the capability of the PSS method for improving the power system's stability, under small perturbation and large perturbation, the dynamic performance of it was analyzed. The performance of the fuzzy logic-based PSS was thoroughly analyzed for comprehending its capacity with the CPSS method using optimized parameters generated from the phase of compensation technique.

During the nominal operating condition, A small perturbation of step increase in mechanical torque was established. The dynamic responses of the proposed PSS were comparatively analyzed with the CPSS. The proposed PSS generated lower peak over-shoots and damped out low frequency oscillations vastly compared to the CPSS approach which depicted in Fig. 5 and 6 . The proposed PSS has a better damped response in overall.

FLPSS tuned PSO with the review of the optimal parameters:

$$
K p=1,4120 \quad K w=0,9592 \quad K u=7,5346
$$
5.

show results very fast reduction as shown in the figure

When compared between FLPSS and CPSS will have the ability to reduce low frequency oscillations more quickly. It as shown in Figure 6.

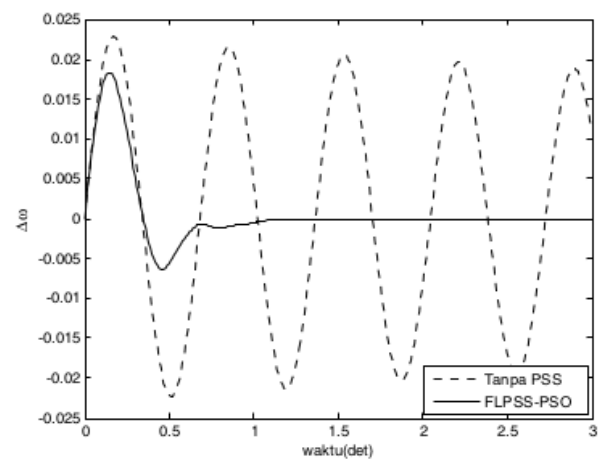

Fig. 5. Response to input torque $\Delta \mathrm{T}_{\mathrm{m}}=1 \mathrm{pu}$

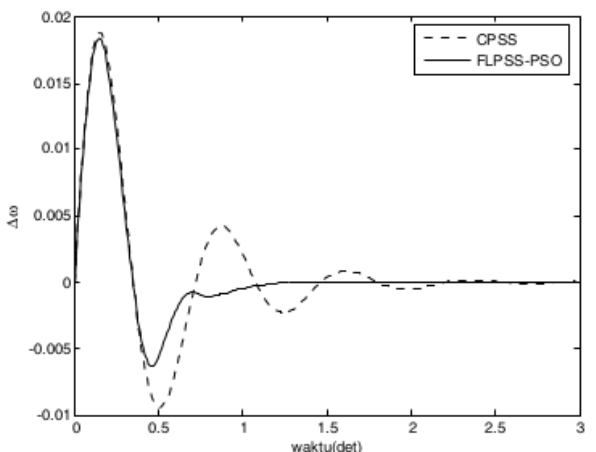

Fig. 6. Response to input torque $\Delta \mathrm{T}_{\mathrm{m}}=1 \mathrm{pu} \Delta \mathrm{T}_{\mathrm{m}}=1$ pu with Conventional PSS and FLPSS-PSO

The simulation results have shown that the fuzzy logic 
controller PSS tuned proposed PSO can be an alternative to dealing with electric power system oscillations. This is shown on the attenuation performed by the controller compared to a system without PSS and PSS with conventional tuned. Selection K and KP through PSO to 30 operations at PSS Fuzzy controller based on input power and speed deviation will produce varying oscillation damping.

\section{CONCLUSION}

The proposed design of optimal PSS controller based on fuzzy showed an effective results and optimal damping so that PSS could become be a stabilizer to handle damping oscillations in electrical power systems. PSS is using Fuzzy controller parameters $K_{p}, K_{\omega}$ and $K_{u}$ performed using PSO with the fitness function in the form of index performance. Indeed, through this election actually can produce oscillation damping speed deviation highly effective and optimal. The simulation results of the electric power system of single-rail engine has demonstrated the ability infinite optimal stabilizer for damping low frequency oscillations and become an alternative stabilizer.

\section{REFERENCES}

[1]. Prabha Kundur. (1994)."Power System Stability and Control". McGraw-Hill, New York, Ch.12. pp.817822.

[2]. Padiyar, (2008),"Power System Dynamics Stability and Control", BISA Publications, Hyderabad, Ch.8. pp 257-294.

[3]. Larsen and D. A. Swann. (1981). "Applying Power System Stabilizers". Part I- III, IEEE Trans. PAS Vol. 100. no 6. pp 3017-3046.

[4]. Ruhua You, Hassan J.E, and M. Hasyem N (2002). "An Online Adaptive Neuro-Fuzzy Power System Stabilizer for Multimachine System", IEEE Transaction On Power System Vol-18, Febuary,

[5]. El-Hawary. M. (1998), Electric Power Applications of Fuzzy Systems, IEEE Press, New York.

[6]. Tang K. S., Kim Fung Man, Guanrong Chen, and Sam Kwong, 2001," An Optimal Fuzzy PID Controller", IEEE Transactions On Industrial Electronics, Vol. 48, No. 4, August 2001.

[7]. J. Kennedy, R. Eberhart. (1995). "Particle swarm optimization," Proc. IEEE Intl. Conf. Neural Networks. 4, Nov/Dec . pp 1942-1948.

[8]. Ali T. Al-Awami etc, (2005). "Simultaneous Stabilization Of Power Systems Equipped With Unified Power Flow Controller Using Particle Swarm", 15th PSCC, Liege, 22-26 August 2005

[9]. Jun-Yi Cao and Bing-Gang Cao. (2006) "Design of Fractional Order Controller Based on Particle Swarm Optimization", International Journal of Control, Automation, and Systems. vol. 4, no. 6, pp. 775-781, December.

[10]. Jaco F. Schuttle. (2005). "Evaluation of a Particle Swarm Algorithm For Biomechanical Optimization", Journal of Biomechanical Engineering June, Vol. 127 $/ 465$. 
PSS Design Based on Fuzzy Controller with Particle Swarm Optimization Tuning

ORIGINALITY REPORT

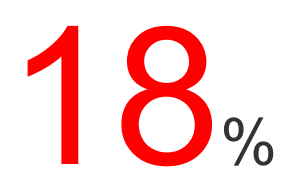

SIMILARITY INDEX
$21 \%$

INTERNET SOURCES
$17 \%$

PUBLICATIONS
$14 \%$

STUDENT PAPERS

PRIMARY SOURCES

1 www.stanford.edu Internet Source

2 www.coursehero.com

\section{3} www.daaam.info

\section{Student Paper}

Exclude quotes

Exclude bibliography
On

On
Exclude matches

$<2 \%$ 